\title{
Cusp Singularity-Based Bistability Criterion for Geometrically Nonlinear Structures
}

\author{
Larry A. Danso and Eduard G. Karpov* \\ Department of Civil and Materials Engineering, University of Illinois at Chicago, \\ 842 West Taylor St., M/C 246, Chicago, Illinois 60607, USA \\ *Corresponding Author: ekarpov@uic.edu
}

\begin{abstract}
This letter introduces a method of analyzing multi-stable truss structures and unit cells of engineered materials using the third order derivative of the formulated system's potential energy function. The method can determine systematically all the cusp point singularities arising at the onset of bistability, and therefore identify the regions of hysteretic superelasticity and superplasticity from the usual geometrical nonlinearity in the solution space. The ability to design these behaviors is a great advantage that is highlighted with the example analysis of a bistable plane truss and a tetrahedral unit cell of a 3D lattice structure.
\end{abstract}

Keywords: bistability, geometrically nonlinear structures, engineered materials

\section{INTRODUCTION}

In a century where the need for smart materials and structures has been discussed extensively, the truss systems are of interest for both engineers and material scientists alike because of their adaptivity for a microstructure or an architectural piece for a controlled and an optimized design. Many truss systems have been studied [1-3] and observed to switch between two different states of equilibrium under an applied load and this is exhibited by a snap-through action. The application of the multiple states of equilibrium that could be achieved with a truss system has been seen in mechanical, electromechanical systems and MEMS. This corresponds with smart applications of buckling induced systems that utilizes the switching actions that accompany the 
buckling instability of a system [4]. Most of the studies done in the area of truss stability have involved numerical or semianalytical computations [5-6]. Some of these works [5,7-8] have shown how geometrical and material nonlinearities affects stability of truss systems and that if properly designed could lead to a desired state of equilibrium. Even with the success of these methods the need for an analytical method to improve universality when analyzing trusses with high geometrical nonlinearity [9] and to fully understand the behavior of these systems including general multistability criteria is recognized [10]. Space trusses analyzed in [8] by numerical methods have shown the possibility of developing a lattice structure that would exhibit multiple states of equilibrium. Interesting applications in smart structures, structural composites and metamaterials can also be envisioned for periodic lattices [11-13], whose behavior can be predicted from the unit cell analysis. The application of lattice structures and elastic networks in materials science and engineering is widespread and therefore it drives our interest as well.

The aim of this letter is to demonstrate an analytical approach for a panoramic analysis of multistable lattice structures and with the relevant concepts in mind. The discussion involves the equilibrium potential energy of the structure with its usual critical point condition and degenerate critical point condition [6,14-16] outlined in most stability studies of lattice structures to generate stability diagrams. However, we introduce a new concept of the cusp curve, the locus of all cusp point singularities in the parametric solution space based on the third order derivative of the potential energy function, which is used as a tool to predict any possible bistable hysteretic behavior of the truss system. It leads essentially to a phase diagram of the system in terms of the corresponding system parameters. Such a phase diagram gives the designer the flexibility to select a desired structural behavior: monostable elasticity, bistable superelasticity, or bistable superplasticity corresponding to the situation when the original configuration can only be recovered by load reversal. The letter illustrates the method using a $2 \mathrm{D}$ lattice first, and then proceeds to a $3 \mathrm{D}$ tetrahedral truss example.

\section{ANALYSIS OF MULTI-STABLE STRUCTURES}

Throughout our analysis we have assumed that the lattice structure is linearly elastic, undergoes moderate axial strains $(<0.05)$ and large nodal displacements leading to a geometrical 
nonlinearity. In our analysis we would like to eliminate the possibility of buckling instability and so the assumption of very high Euler load is made [14]. We therefore apply the principle of stationary potential energy to get the total potential energy and applying the critical points condition $\left(\frac{d \Pi}{d u}=0\right)$ for the system we arrive at the system equilibrium equation that is analyzed for the stable states of our lattice structure. The total potential energy, $\Pi$, of our system is defined as the total strain energy, $U$, stored in the bars less the potential energy of the external loads, $V$,

$$
\Pi=U-V
$$

The strain energy stored in each bar or elastic link could be expressed as

$$
U=\frac{E A(l-L)^{2}}{2 L}
$$

with $E, A, L$ and $l$ denoting the Young's modulus, cross-sectional area, relaxed and deformed lengths of the bar, respectively. Since $E A / L$ represents the stiffness, $k$, of the bar and Cauchy strain, $\varepsilon_{c}=(l-L) / L$, where $l$ is the deformed length, Eqn. 2 can be written as

$$
U=\frac{1}{2} k L^{2} \varepsilon_{c}^{2}
$$

As would be seen in the foregoing analysis $\varepsilon_{c}$ can be replaced with the Green's strain,

$$
\varepsilon_{G}=\frac{l^{2}-L^{2}}{2 L^{2}}
$$

This is adapted for the lattice elements knowing the relationship between the two could be expressed as $\varepsilon_{G}=\varepsilon_{c}+\frac{1}{2} \varepsilon_{c}{ }^{2}$ [17] and hence the difference in strain value is insignificant $(<0.3 \%)$ on the assumption of moderate axial strains.

The potential energy of the applied load, $F$, acting against the strain energy in the bars is expressed as $V=F u$ with $u$ denoting the nodal displacement under the load.

The basic principle in our analysis would be to find the equilibrium equation of the truss system and based on the control parameters present, prepare the stability and phase diagrams that would be used to control and design the bistable superelastic or superplastic response of the structure. 


\subsection{Plane Truss Analysis}

The first truss to be analyzed is the five-bar plane truss in Figure 1, a more complicated case of the von Mises truss whose stability analysis is well documented [15,18-19]. The top bars are assumed to have the same stiffness and similar for the bottom bars as shown.

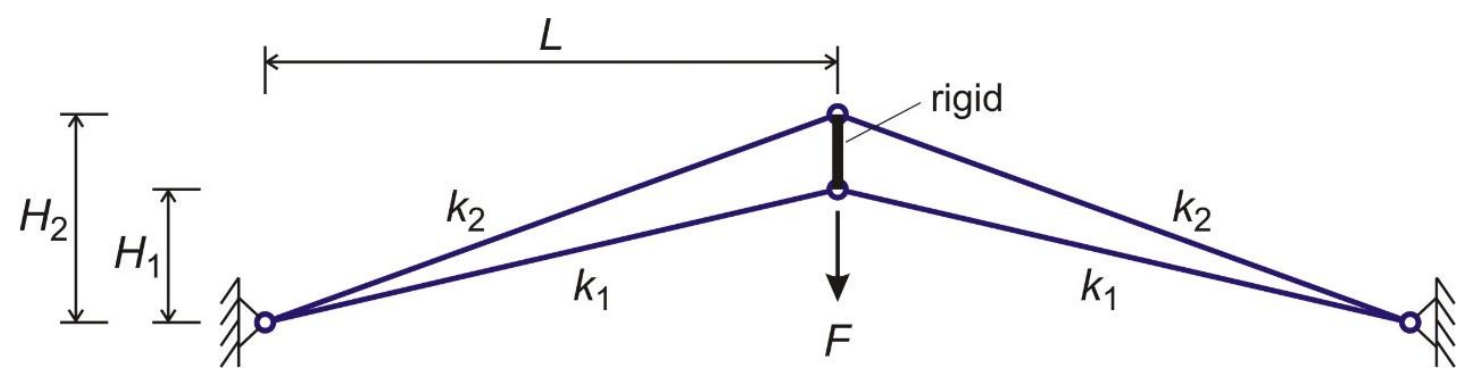

Fig. 1 Bistable five-bar plane truss

The Green's strain from Eqn. 4 for the bars $k_{1}$ and $k_{2}$ are obtained as

$$
\begin{gathered}
\varepsilon_{1}=\frac{u^{2}-2 H_{1} u}{2\left(L^{2}+H_{1}{ }^{2}\right)} \\
\varepsilon_{2}=\frac{u^{2}-2 H_{2} u}{2\left(L^{2}+H_{2}{ }^{2}\right)}
\end{gathered}
$$

Therefore the strain energy stored in the bars from Eqn. 3 are expressed as

$$
\begin{aligned}
& \pi_{1}=\frac{1}{8} \frac{k_{1}}{\left(L^{2}+H_{1}^{2}\right)}\left[u^{2}-2 H_{1} u\right]^{2} \\
& \pi_{2}=\frac{1}{8} \frac{k_{2}}{\left(L^{2}+H_{2}^{2}\right)}\left[u^{2}-2 H_{2} u\right]^{2}
\end{aligned}
$$

The total potential energy, Eqn. 1, of this structure reads

$$
\Pi=\frac{k_{1}}{4\left(L^{2}+H_{1}^{2}\right)}\left[u^{2}-2 H_{1} u\right]^{2}+\frac{k_{2}}{4\left(L^{2}+H_{2}^{2}\right)}\left[u^{2}-2 H_{2} u\right]^{2}-F u
$$

Therefore applying the principle of equilibrium potential energy we obtain the governing equilibrium equation: 


$$
\frac{d \Pi}{d u}=\frac{k_{1}}{\left(L^{2}+H_{1}^{2}\right)}\left(u^{2}-2 H_{1} u\right)\left(u-H_{1}\right)+\frac{k_{2}}{\left(L^{2}+H_{2}^{2}\right)}\left(u^{2}-2 H_{2} u\right)\left(u-H_{2}\right)-F=0
$$

To simplify the analysis we minimize the number of design variables in Eqn.10 by writing it in a reduced dimensionless form. The dimensionless force $f$ and displacement $x$ can be introduced as

$$
x=\frac{u}{H_{1}} \quad f=\frac{F}{H_{1}} \frac{\left(L^{2}+H_{1}^{2}\right)\left(L^{2}+H_{2}^{2}\right)}{\left(L^{2}+H_{1}^{2}\right) k_{2}+\left(L^{2}+H_{2}^{2}\right) k_{1}}
$$

Then we may introduce only two dimensional system parameters, $a$ and $b$,

$$
a=\frac{3}{H_{1}} \frac{\left(L^{2}+H_{1}^{2}\right) H_{2} k_{2}+\left(L^{2}+H_{2}^{2}\right) H_{1} k_{1}}{\left(L^{2}+H_{1}^{2}\right) k_{2}+\left(L^{2}+H_{2}^{2}\right) k_{1}} \quad b=\frac{2}{H_{1}^{2}} \frac{\left(L^{2}+H_{1}^{2}\right) H_{2}^{2} k_{2}+\left(L^{2}+H_{2}^{2}\right) H_{1}^{2} k_{1}}{\left(L^{2}+H_{1}^{2}\right) k_{2}+\left(L^{2}+H_{2}^{2}\right) k_{1}}
$$

and write the following dimensionless form of the equation of equilibrium for our truss structure:

$$
f=x^{3}-a x^{2}+b x
$$

\subsection{Stability Diagrams}

At this point we have been successful in achieving a simplified equilibrium equation in Eqn. 13 which can serve as a simple tool to understand the multiple states of equilibrium of the truss structure. It will also enable us to develop relationships between the various control parameters $(f, a, b)$ of the structure, using the stability diagram concept. A stability diagram is a plot of bifurcation curves comprised of all possible bifurcation points that may exist for the system in the $(f, a, b)$ space. This concept seen in catastrophe theory [16,20-21] and thus shows how the control parameter $f$ and the system parameters $a$ and $b$ affect the states of equilibrium. We obtain the sought stability diagram by projecting the $3 \mathrm{D}$ solution space of $\frac{d \prod}{d u}$ of Eqn. 13 into the $(f, a)$ or $(f, b)$ plane, depending on which system parameter is fixed. Figure 2 shows the topology of the solution space when $a$ is held constant and we make an orthogonal projection toward the $(f, b)$ the plane. The stability diagram could also be obtained directly by plotting the functions that describe the bifurcation curves. 


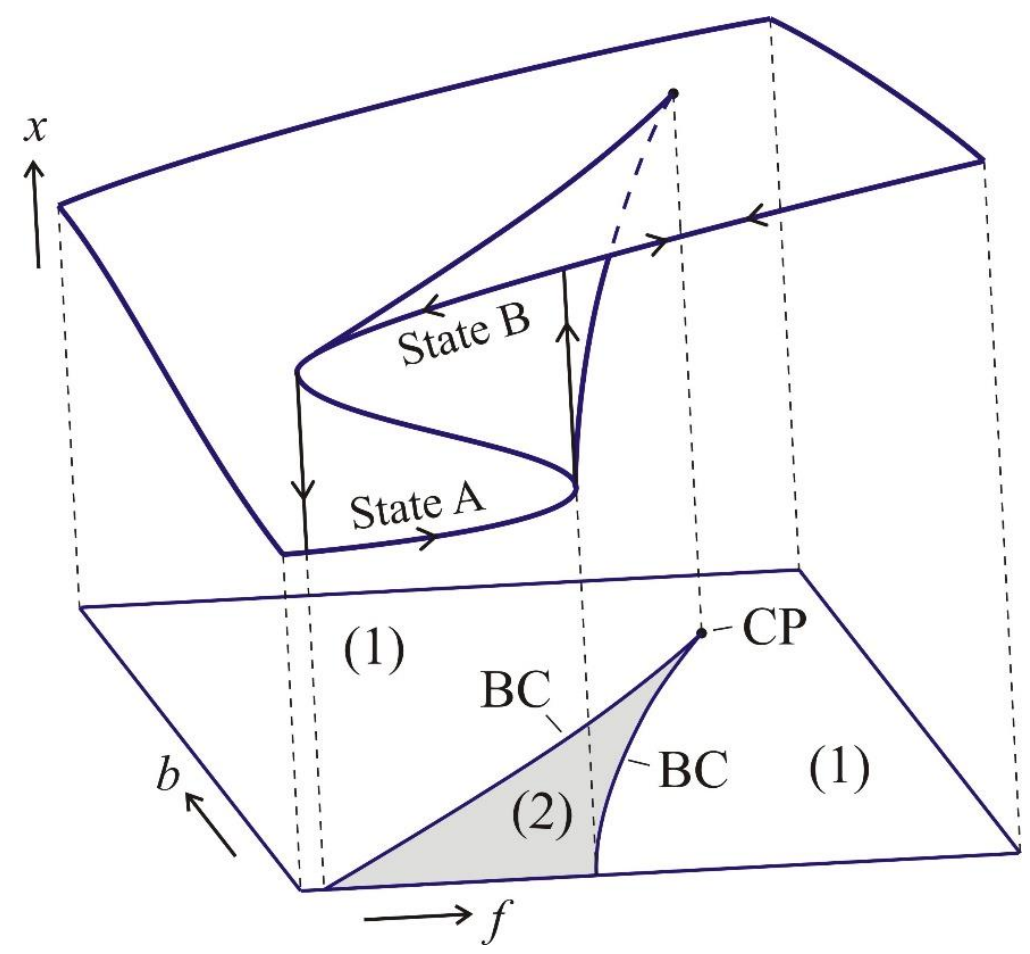

Fig. 2 A three-dimensional view of the folded solution space (13) at constant $a$, and its bifurcation set projection onto the $f b$-plane; $\mathrm{CP}$ - the cusp point; $\mathrm{BC}-$ bifurcation curves, the locus of all existing ( $a b$-parameterized) bifurcation points; (1) - region of unique solutions; (2) - region of two stable solutions. For a value $b$ fixed within the region (2), the system experiences a jump in response (switching between two stable equilibrium states) when an increasing/decreasing load crosses the right/left bifurcation curve, producing a mechanical hysteresis.

The functions describing the bifurcation curves are obtained by solving the degenerate condition, $\frac{d^{2} \Pi}{d u^{2}}=0$, for solutions to the system state variable $y$ which is given by Eqn.14 and then substituting each solution obtained into Eqn. 13:

$$
x=\frac{a \pm \sqrt{a^{2}-3 b}}{3}
$$



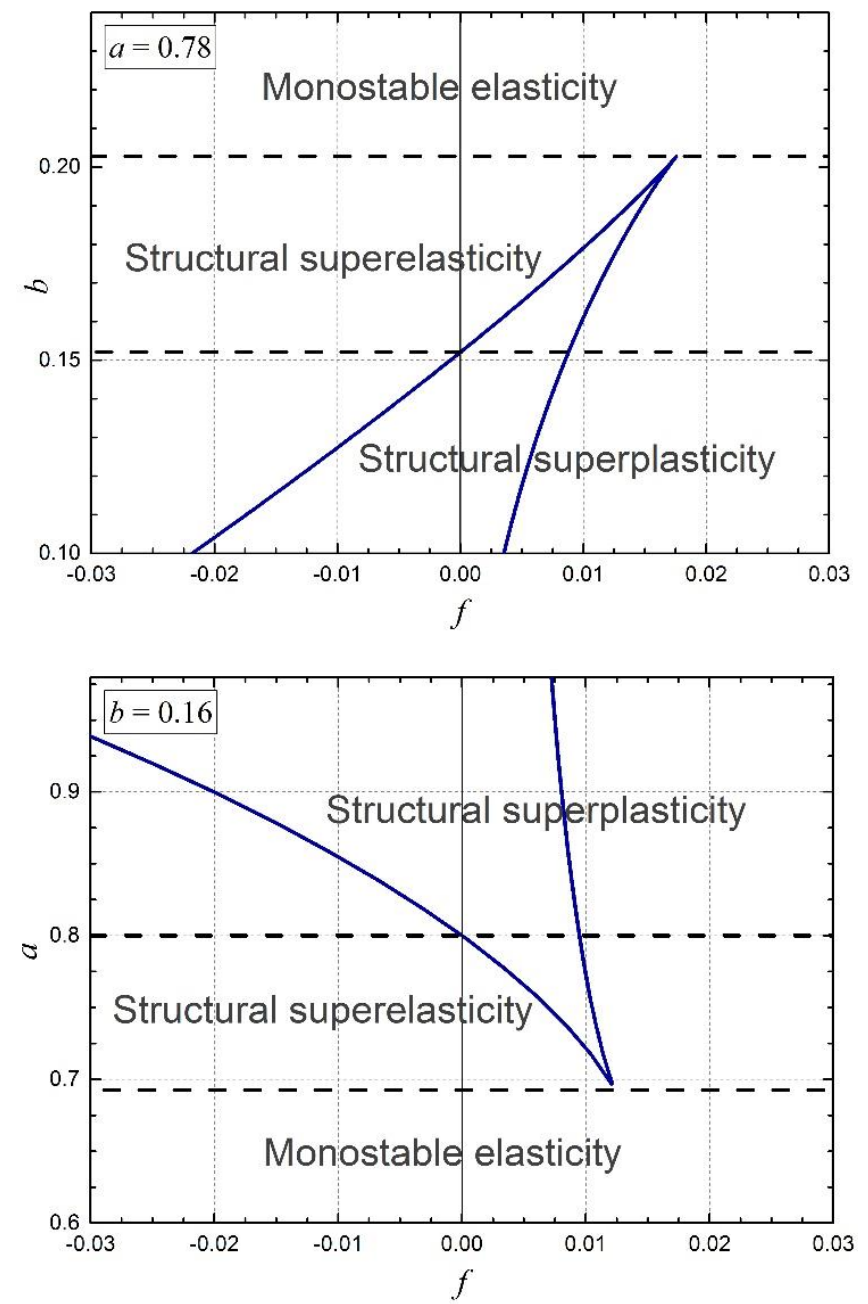

Fig. 3 Stability diagrams for the parameter $b$ versus the dimensionless force $f$ at $a=0.78$ (top), and for the parameter $a$ versus $f$ at $b=0.16$ (bottom). Structural superelasticity is the region where the system can experience a reversible transition between the two stable states with load application and removal. In the structural superplasticity region, the system also switches between two stable states but there is a residual deformation when the load is removed $(f=0)$, and a full recovery is only possible with a load reversal.

Eliminating the state variable $x$ with this step we obtain two functions in terms of only the control and system variables $f, a, b$ which describe the set of bifurcation curves for the truss system. Hence, the stability diagrams, Figure 3, are obtained by fixing one of the system variables, either $a$ or $b$ in the functions obtained for the bifurcation curves to a desirable constant value, and 
plotting the other system parameter as a function of the dimensionless force $f$. Each point on the bifurcations curves represents a saddle-node bifurcation with the force $f$ as the control parameter, except for the point at the cusp of the plots in Figure 3. This cusp point (CP) represent a higher order singularity where a supercritical pitchfork bifurcation may occur on the parameter plane $(f, b)$ or $(f, a)$ in the direction tangential to the cusp. Consequently, the areas between the two lines represent a region where two stable and one unstable solution of the governing equilibrium equation exists, and only one (stable) solution exists on all other areas of the stability diagrams.

Since the parameter $f$ represents the external load, a hysteretic superelastic or superplastic type response to a cyclic load is therefore possible for a range of parameters $a$ and $b$. Typical forms of these responses, (SE) and (SP), are shown in Figure 4 in comparison to the usual nonlinear response, when one of the parameters ( $a$ or $b$ ) are located in the monostable elasticity (ME) region.

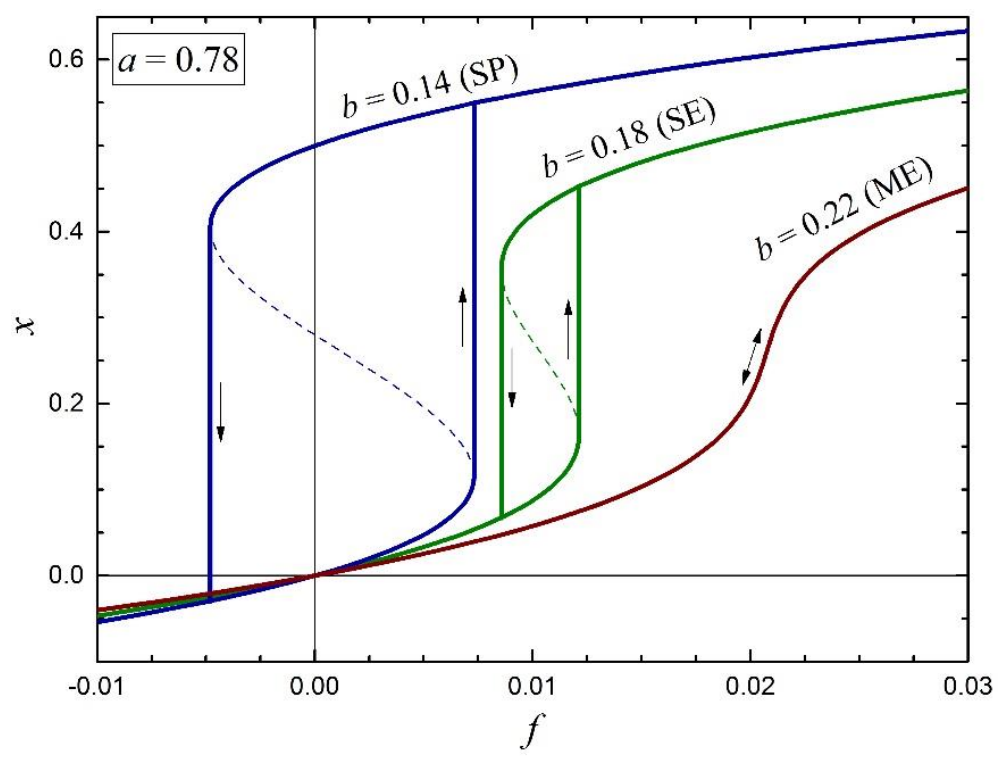

Fig. 4 Three types of structural responses governed by the equilibrium equation (13): structural superplasticity (SP), structural superelasticity (SE), and the usual monostable elastic (ME) behavior. The inner dash lines represent the unstable equilibrium configurations. 
As an example, for a five-bar truss with a set of design parameters chosen as $L=0.5 \mathrm{~m}, H_{1}=$ $0.0822 \mathrm{~m}, H_{2}=0.020 \mathrm{~m}, k_{1}=2.32 \times 10^{5} \mathrm{~N} / \mathrm{m}$ and $k_{2}=1.0 \times 10^{7} \mathrm{~N} / \mathrm{m}$, see Figure 1 (bar $k_{1}$ is above $k_{2}$ in this example), we obtain $a=0.78$ and $b=0.16$ as the dimensionless system parameters. The both stability diagrams of Figure 3 indicate that such a truss will experience forward and reverse superelastic transition at the (critical) loads of 0.00986 and 0.00304 dimensionless units, or 224 and $69 \mathrm{~N}$, respectively. The first diagram shows the case when $a$ is fixed and the second when $b$ is fixed. In both plots we encounter a single cusp point at the onset of bistability, which could be predicted using a mathematical condition proposed in the following section.

\subsection{Cusp Curve and System Phase Diagram}

As can be seen from Figure 2, the solution space of the five-bar structure contains a higher order singularity, the cusp point (CP). Physically, such a point represent the onset of bistability in the system; for example, the $b$-coordinate of the cusp point in Figure 3 (top) represents a critical value of this system parameter, below which a hysteretic mechanical response becomes possible.

In the design of our system, the stability curves could be created for all possible values of one particular system parameter and these plots would help control and optimize the desired system parameter. To simplify this process we develop the concept of the cusp curve, a plot of only cusp points in the $a b$-plane, which separates the entire region of hysteretic (bistable) elasticity from the usual (monostable) geometrically nonlinear elasticity.

We develop the cusp curve by solving simultaneously the condition $\frac{d^{3} \Pi}{d u^{3}}=0$ (leading to $x=a / 3$ ) with the degenerate condition, $\frac{d^{2} \Pi}{d u^{2}}=0$, and the equilibrium equation, Eqn. 13. This gives an expression in terms of only the control parameters $a$ and $b$, which is solved for $b$ in Eqn. 15. In order to separate further the most interesting region of structural superelasticity from the region of superplasticity (with a residual deformation at force removal), the force condition $f=0$ by Eqn. 13 is solved together with the degenerate condition to give the Eqn. 16.

$$
b=\frac{a^{2}}{3}
$$




$$
b=\frac{a^{2}}{4}
$$

These two functions are then plotted as shown in Figure 5 to represent a phase diagram of the system.

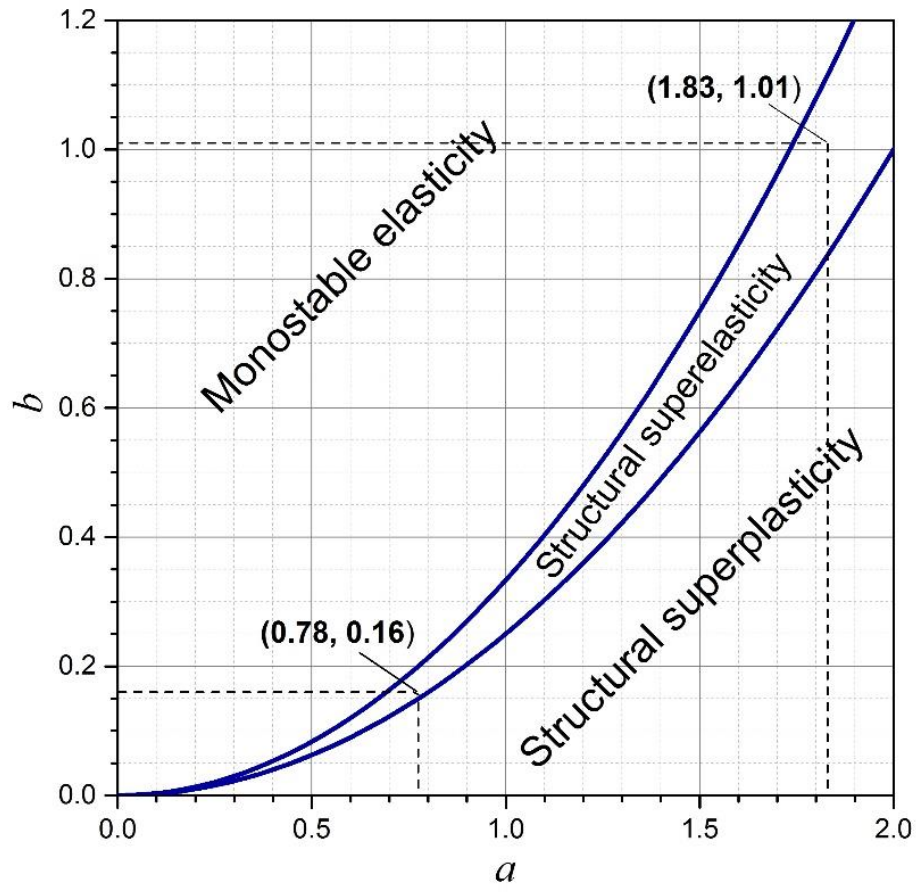

Fig. 5 Phase diagram of elastic systems of the type $f=x^{3}-a x^{2}+b x$, showing the regions of principal mechanical behavior. The line from Eqn. 15 divides the regions of structural monostability and bistability, and the Eqn. 16 line subdivides the bistability into the structural superelasticity and structural superplasticity.

To illustrate the use of the phase diagram we consider the design case used above and locate the obtained system parameters $a=0.78$ and $b=0.16$ on the graph. As can be seen, this design is within the structural superelastic region, being in agreement with the Figure 3 stability diagrams.

Thus, the desired stability behavior could be achieved by manipulating the original design parameters in Figure 1, and identifying the position of a resultant pair of system parameters $a$ and 
$b$ on the phase diagram. From Figure 5, the region of structural superelasticity is a narrow band which represents somewhat a rare case of achieving such a behavior in a design. Therefore, one may see how important a phase diagram, using the cusp point and zero-force conditions, could be for a design process.

We note that utility of the Green's strain measure (4) in the potential energy form (3) is the main approximation of the method enabling the analytical stability analysis of sections 2.2 and 2.3. Since the Green's strain and Cauchy stress tensors are not work conjugate, this approximation should be used in a cautious manner. The method can be quick and reliable in identifying a possibility to achieve bistability as in principle; however, accuracy of the phase diagram boundaries will diminish with an increase of maximal strain in members using the Green's strain measure. This latter factor can serve as an indicator of the method's applicability: the maximal strain in those members using the Green's strain measure for their potential energy form (3) should not exceed 0.04-0.05 during the entire load-unload cycle, including the state-switching regimes,

due to the relationship $\varepsilon_{G}=\varepsilon_{c}+\frac{1}{2} \varepsilon_{c}{ }^{2}$. Furthermore, validation of the method for all types of structures can be performed in a straightforward manner by using the Cauchy strain for all structural members in the total potential energy (9) and running a quasistatic energy minimization procedure to get a numerical load-deformation curve of Fig.4 type. Results of our various validations runs indicate that the maximal error for all state and system parameters, including the critical loads, is on the order of the mismatch between $\varepsilon_{G}$ and $\varepsilon_{c}$, provided that the Green's strain in structural members remains in the moderate range $\left(\varepsilon_{G}<0.05\right)$ for an entire load-unload cycle. For the design example discussed in the end of section 2.2, this mismatch does not exceed $0.1 \%$ over the load cycle shown in Figure 4.

\subsection{Tetrahedral Structure Analysis}

In this section we adapt the analytical procedure of the five-bar truss to analyze the tetrahedral 3D lattice structure of Figure 6 for variation in the stiffness of its outer shell and inner core elements. The stability analysis of this unit cell can provide the basis for generalizing the behavior of various multistable periodic structures under the application of external loads. 
The strain energy in the bars is written as below using the fact that the base triangle of the modified tetrahedral truss model is equilateral, the assumption that the length of the exterior bars are equal and in a similar manner all the inclined interior bars are of equal length. The top node, where the force is applied is constrained to move vertically (nodal displacement $v$ ), and the three bottom nodes can only move in the base plane of the tetrahedron (nodal displacement $u$ ). The stiffness of each bar is as shown on Figure 6. Then, their corresponding strain energies are:

$$
\begin{aligned}
& \pi_{1}=\frac{k_{1}}{24 B^{2}}\left(u^{2}+2 B u+v^{2}-2 \sqrt{2} B v\right)^{2} \\
& \pi_{2}=\frac{k_{2}}{8\left(H^{2}+B^{2}\right)}\left(u^{2}+2 B u+v^{2}-2 H v\right)^{2} \\
& \pi_{3}=\frac{k_{3}}{8 B^{2}}\left(u^{2}+2 B u\right)^{2}
\end{aligned}
$$
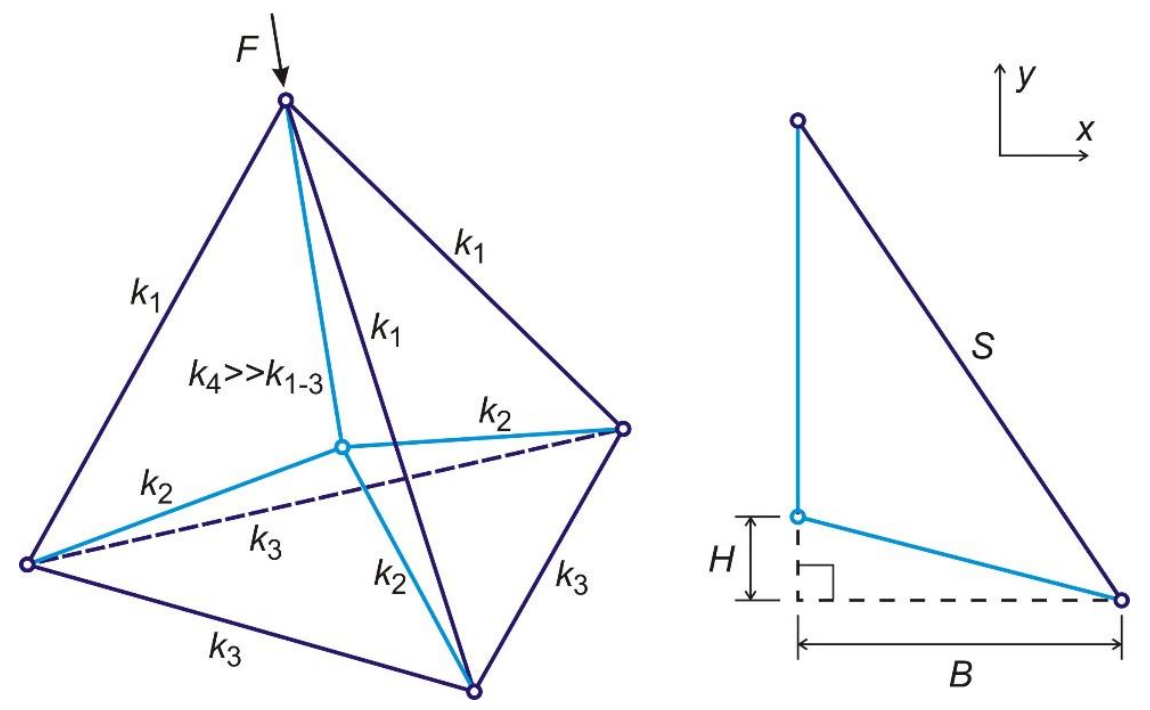

Fig. 6 Tetrahedral lattice cell with an inner core: a side view of structure showing the direction of applied load and bars stiffnesses, and a sectional view of the $x y$ plane from the vertical line of symmetry to a base node $(S=\sqrt{3} B)$.

The total strain energy due to the nine bars and the external load reads 


$$
\Pi=\frac{k_{1}}{8 B^{2}}\left(u^{2}+2 B u+v^{2}-2 \sqrt{2} B v\right)^{2}+\frac{3 k_{2}}{8\left(H^{2}+B^{2}\right)}\left(u^{2}+2 B u+v^{2}-2 H v\right)^{2}+\frac{9 k_{3}}{8\left(B^{2}\right)}\left(u^{2}+2 B u\right)^{2}-F v
$$

The two equilibrium equations derived from Eqn. 18 are

$$
\begin{aligned}
& 0=\frac{d \Pi}{d v}=\frac{k_{1}}{2 B^{2}}\left(u^{2}+2 B u+v^{2}-2 \sqrt{2} B v\right)(v-\sqrt{2} B)+\frac{3 k_{2}}{2\left(H^{2}+B^{2}\right)}\left(u^{2}+2 B u+v^{2}-2 H v\right)(v-H)-F \\
& 0=\frac{d \Pi}{d u}=\frac{k_{1}}{2 B^{2}}\left(u^{2}+2 B u+v^{2}-2 \sqrt{2} B v\right)+\frac{3 k_{2}}{2\left(H^{2}+B^{2}\right)}\left(u^{2}+2 B u+v^{2}-2 H v\right)+\frac{9 k_{3}}{2\left(B^{2}\right)}\left(u^{2}+2 B u\right)
\end{aligned}
$$

These equations can be rewritten in the dimensionless forms as

$$
\begin{gathered}
g=\left(x^{2}+2 x+y^{2}-2 \sqrt{2} y\right)(y-\sqrt{2})+\alpha\left(x^{2}+2 x+y^{2}-2 h y\right)(y-h) \\
0=\left(x^{2}+2 x+y^{2}-2 \sqrt{2} y\right)+\alpha\left(x^{2}+2 x+y^{2}-2 h y\right)+\beta\left(x^{2}+2 x\right) \\
g=\frac{F}{k_{1} B}, \quad \alpha=\frac{3 k_{2}}{k_{1}\left(h^{2}+1\right)}, \beta=\frac{9 k_{3}}{k_{1}}, \quad y=\frac{v}{B}, \quad x=\frac{u}{B}
\end{gathered}
$$

To obtain a single expression in terms of the state variable $y$ only, we derive the expression in Eqn. 24 from Eqn. 22 and substitute the result into Eqn. 21:

$$
\begin{gathered}
\left(x^{2}+2 x\right)=-\gamma\left(y^{2}-2 \sqrt{2} y\right)-\alpha \gamma\left(y^{2}-2 h y\right) \\
\gamma=\frac{1}{\alpha+\beta+1} \\
g=\left[(1-\gamma)\left(y^{2}-2 \sqrt{2} y\right)-\alpha \gamma\left(y^{2}-2 h y\right)\right](y-\sqrt{2})+\alpha\left[(1-\alpha \gamma)\left(y^{2}-2 h y\right)-\gamma\left(y^{2}-2 \sqrt{2} y\right)\right](y-h)
\end{gathered}
$$

Grouping and simplifying the terms we obtain a similar cubic force equilibrium equation, as in Eqn. 13, although with a new dimensional force $f$ and new coefficients $a$ and $b$ :

$$
\begin{aligned}
& f=y^{3}-a y^{2}+b y \\
& f=\frac{g}{1+\alpha-\gamma-2 \alpha \gamma-\alpha^{2} \gamma}, \quad a=-\frac{3 \sqrt{2}(\gamma+\alpha \gamma-1)+3 h\left(\alpha \gamma-\alpha+\alpha^{2} \gamma\right)}{1+\alpha-\gamma-2 \alpha \gamma-\alpha^{2} \gamma}, \quad b=\frac{4(1-\gamma)-4 \sqrt{2} \alpha \gamma h+2 h^{2}\left(\alpha-\alpha^{2} \gamma\right)}{1+\alpha-\gamma-2 \alpha \gamma-\alpha^{2} \gamma}
\end{aligned}
$$

It is useful to note that this equilibrium equation corresponds to a generic nondimensional potential energy form

$$
U(y, f, a, b)=y^{4} / 4-a y^{3} / 3+b y^{2} / 2-f y
$$


Having derived the governing equilibrium equation, Eqn. 27, stability diagrams could be created using the same procedure outlined above for the four bar truss. Since both structures produced the same form of the system equilibrium equation, the phase diagram in Figure 5 would be the same for the modified tetrahedral truss and so it is referenced here.

In the case of a specific tetrahedral truss with the following design parameters, $B=0.1 \mathrm{~m}, H=$ $0.05 \mathrm{~m}, k_{1}=10^{4} \mathrm{~N} / \mathrm{m}, k_{2}=3 \cdot 10^{4} \mathrm{~N} / \mathrm{m}$ and $k_{3}=2 \cdot 10^{4} \mathrm{~N} / \mathrm{m}$, we obtain $a=1.83$ and $b=$ 1.01 as the system parameters, and as shown on Figure 5, the system is positioned in the structural superelasticity region. Undertaking reverse designs using the values within the superelastic region, it should be noted that the stiffness coefficients must remain positive in all cases. One can determine the critical loads at which the tetrahedral truss will experience the forward and reverse superelastic transitions from a new set of stability diagrams similar to those in Figure 3 plotted for the $a$ and $b$ values found here, or by solving simultaneously the Eqs. 13 and 14. These loads are 0.175 and 0.149 dimensionless units, or 986 and $839 \mathrm{~N}$, respectively.
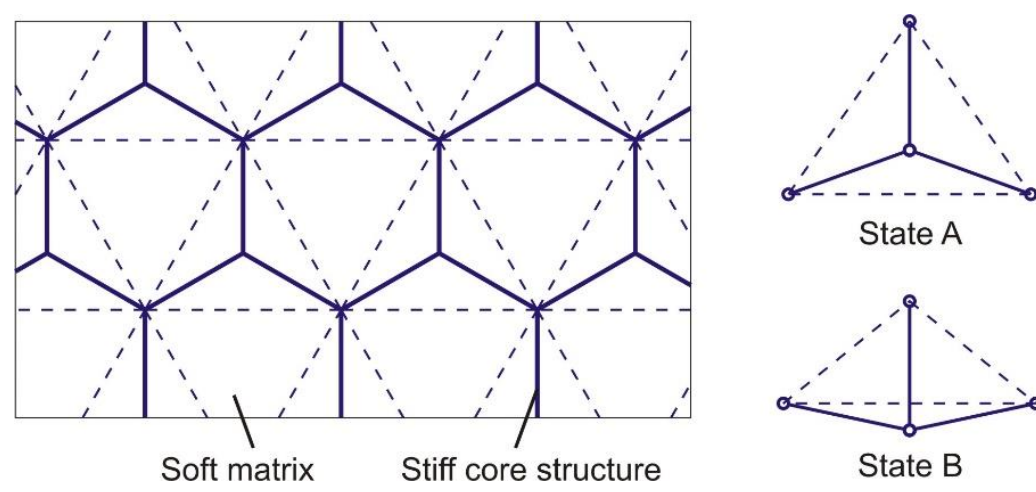

State A

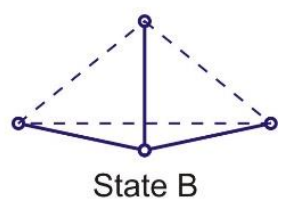

Fig. 7 Periodic structure and the bistable unit cell of an engineered material composed of a stiff reinforcement core immersed in a soft continuum matrix. Dash lines represent effective interactions between the nearest nodes due to the matrix material.

\section{CONCLUSIONS}

This letter has shown the predictive power of the stability diagrams when combined with the phase diagram, obtained on the basis of cusp point singularity parameterization in the solution space, in 
analyzing potentially bistable structural systems. The truss systems analyzed have shown that the governing equilibrium equation for deformation under a load for some periodic lattice trusses could be classified into a basic generalized form as shown for the two trusses analyzed. This form can be regarded as a canonical equation of equilibrium of geometrically nonlinear trusses governed by the Green's strain measure with one independent degree of freedom. It has been shown that for certain truss systems the potential of the superelastic behavior could be rare due to the narrow band of the region on the phase diagram. Nonetheless, the phase diagram shows all the possible parameters values that would lead to the structural superelasticity. Overall, the ability to design standalone bistable trusses and unit cells of a periodic materials with one independent degree of freedom has been understood using the analytical procedure shown in this letter.

In the future, it would certainly be interesting to expand this methodology to the collective behavior of periodic structures and composite materials with bistable unit cells. Such a material would be a $2^{N}$-stable mechanical systems ( $N$ is the number of unit cells) with interesting global properties for many potential applications including impact absorbers, compliant and resilient metamaterials, and shape-memory enabled polymorphic structures. The honeycomb-foam structure of Figure 7 is an example of an engineered material with a soft continuum matrix surrounding a stiff reinforcing core of hexagonal elements. The interaction between the core structure and the matrix material could therefore be idealized by the unit cell shown in the figure whose analysis would follow the same method above. Likewise, many multistable 3D lattices and frameworks can be analyzed on the basis of the bistable tetrahedron-shaped unit cell discussed in section 2.4. The role and utility of periodic boundary conditions for the $2^{N}$-stable periodic structures should also be investigated. Furthermore, structural systems and materials featuring unit cells with two or more independent degrees of freedom are worth of separate studies. In particular, bistable unit cells with one additional (internal) degree of freedom were shown recently [23-24] to provide opportunities for mechanical metamaterials [23-27] with negative compressibility properties.

\section{ACKNOWLEDGEMENTS}

This work is supported in part by the U.S. National Science Foundation via Grant \#1634577. 


\section{REFERENCES}

[1] Cai J. G., Xu Y. X., Feng J. and Zhang J., 2011. Mechanical Behavior of Bistable Struts. Proc. IMech Vol. 226 Part C: J. Mechanical Engineering Science

[2] Plante J.S. and Santer M., 2005. Compliant Bistable Dielectric Elastomer Actuators For Binary Mechatronic Systems, Proceedings Of IDECT/CIE 2005 ASME Mechanism And Robotics Conference 24-28 September 2005 Long Beach, California, USA

[3] Kinoshita T., Oshaki M., 2009. Synthesis of Bistable Compliant Structures from Truss Mechansims, Journal of Computational Science and Technology 3(2), 417-425.

[4] Hu N. and Burgueno R., 2015. Buckling-induced smart applications: recent advances and trends, Smart Materials and Structures 24: 063001

[5] Wang S. T., Blandford G. E. and Hill C. D., 1988. Nonlinear Analysis of Steel Space Trusses, 2010, International Specialty Conference on Cold Formed Steel Structures, Paper 2

[6] Greco M. and Venturini W. S., 2006. Stability Analysis of Three-dimensional Trusses, Latin American Journal of Solids and Structures 3: 325-344

[7] Noemi F. and Adnan I., 2013. Overview of highly flexible, deployable lattice structures used in architecture and civil engineering undergoing large displacements, YBL Journal of Built Environment 1(1): 85-103

[8] Hrinda A.G., 2010. Snap-through Instability Patterns in Truss Structures, NASA Langley Research Centre, AAIA 2010-2611

[9] Shon S., Lee S., Ha J. and Cho C., 2015. Semi- Analytic Solution and Stability of Space Truss Using a Higher-Order Taylor Series, Materials 8: 2400-2414.

[10] Greco M. and Vicente E. R. C., 2009. Analytical Solutions for Geometrically Non-linear Trusses, REM: R. Esc. Minas 62(2): 205-214.

[11] Karpov E.G., Dorofeev D.L. and Stephen N.G., 2002. Characteristic Solutions for the Statics of Repetitive Beam-Like Trusses. International Journal of Mechanical Sciences 44(7): 1363-1379. [12] Karpov E.G., Stephen N.G. and Dorofeev D.L., 2002. On Static Analysis of Finite Repetitive Structures by Discrete Fourier Transform. International Journal of Solids and Structures 39(16): 4291-4310. 
[13] Karpov E.G., 2017. Structural Metamaterials with Saint-Venant Edge Effect Reversal. Acta Materialia 123, 245-254.

[14] Liagro S.S. and Valvo P.S., 2006. Large displacement analysis of elastic pyramidal trusses, International Journal of Solids and Structures 43(16): 4867-4887

[15] Bazant Z. P. and Cedolin L., 1991. Stability of Structures, Oxford University Press, 228-231

[16] Yin J. P., Marsh D. and Duffy J., 1998. Catastrophe analysis of planar three-spring systems. ASME Design Engineering Technical Conferences, DETC 98/MECH-5919

[17] Ivanco V., 2011. Nonlinear Finite Element Analysis: Script of Lectures, HS Wismar

[18] Pecknold D. A., Ghaboussi J. and Healey T. J., 1985. Snap-through and Bifurcation in a Simple Structure, J. Eng. Mech. 111: 909-922

[19] Eremeyev V. A. and Lebedev P., 2006. On the loss stability of von Mises truss with the effect of pseudo-elasticity, Matemáticas XIV(2): 111-118

[20] Gilmore R., 1981. Catastrophe theory for Scientists and Engineers, Dover

[21] Strogatz S. H., 1994. Nonlinear Dynamics and Chaos, Addison-Wesley Publishing Company [22] Loukaides E.G. and Seffen K.A., 2015. Multistable grid and honeycomb shells, International Journal of Solids and Structures

[23] Nicolaou Z.G. and Motter A.E., 2012. Mechanical metamaterials with negative compressibility transitions, Nature Materials 11: 608.

[24] Chen M.L. and Karpov E.G., 2014. Bistability and Thermal Coupling in Elastic Metamaterials with Negative Compressibility. Physical Review E 90: 033201.

[25] Schenk M. and Guest S.D., 2013. Geometry of Miura-Folded Metamaterials. PNAS: 110(9), 3276-3281.

[26] Wei Z.Y., Guo Z.V., Dudte L., Liang H.Y. and Mahadevan L., 2013. Geometric Mechanics of Periodic Pleated Origami. Physical Review Letters 110: 215501.

[27] Silverberg J.L., Evans A.A., McLeod L., Hayward R.C., Hull T., Santangelo C.D. and Cohen I., 2014. Using Origami Design Principles to Fold Reprogrammable Mechanical Metamaterials. Science 345, 647-650. 